\title{
The Performance Evaluation for Personal Identification using Palm print and Face Recognition
}

\author{
Hlaing Htake Khaung Tin \\ Software Technology Department \\ Computer University (Loikaw), KaYah State, Myanmar
}

\begin{abstract}
Biometrics helps to provide the identity of the user based on his/her physiological or behavioural characteristics. The physiological characteristics signifies using human body parts for authentication like fingerprint, iris, ear, palm print, face etc. The behavioural characteristics include action done using body parts like voice, signature and gait etc. Palm print is one of the relatively new physiological biometrics due to its stable and unique characteristics. The rich texture information of palm print offers one of the powerful means in personal identification and verification. Biometric palm print recognizes a person based on the principal lines, wrinkles and ridges on the surface of the palm. These line structures are stable and remain unchanged throughout the life of an individual. The proposed methodology is divided into four steps: image acquisition by a regular webcam, image pre-processing for image normalization, segmentation for biometric extraction and human interpretation. The goals of the face recognition system are 1) to create the fast recognition system for the face database 2) to predict how old the person is and to carry out the face recognition system based on this predicted age 3) to stop underage drinkers from entering bars, prevent minors from purchasing tobacco products from vending machines 4) deny children access to adult Web sites by predicting their age. The main advantage of this system is reduction of searching time and it requires small memory usage. According to the experiment result, this system is an effective age dependent face recognition system. The proposed system has been tested with different images. Experimental tests have proved that the proposed approach is not only robust but also quite efficient.
\end{abstract}

Keywords: Personal Identification, Verification, Biometrics, Palm print

\section{Introduction}

There are two types of palm print recognition research, high resolution and low resolution approaches. High resolution approach employs high resolution images while low resolution approach employs low resolution images. High resolution approach is suitable for forensic applications such as criminal detection [1]. Low resolution images are more suitable for civil and commercial applications such as access control. Generally speaking, high resolution refers to $400 \mathrm{dpi}$ or more and low resolution refers to 150 dip or less.

In high resolution images, researchers can extract ridges, singular points and minutia points as features while in low resolution images, they generally use principal lines, wrinkles and texture. At the beginning of palm print research, the high- resolution approach was the focus [2-3] but almost all current research is focused on the low resolution approach because of the potential applications. In this paper, we concentrate only on the low resolution approach since it is the current focus.

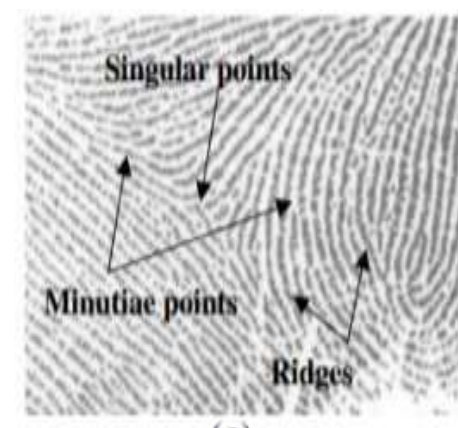

(a)

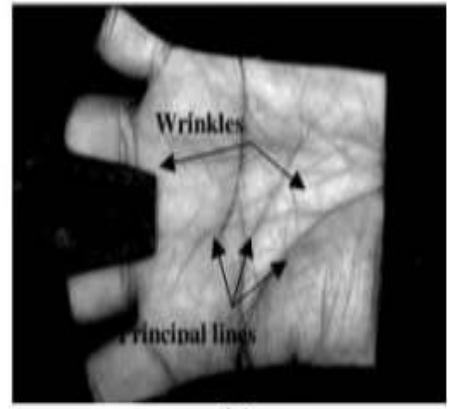

(b)

Fig. 1: Palm print features in (a) a high resolution image and (b) a low resolution image 
For civil and commercial applications, low-resolution palm print images are more suitable than highresolution images because of their smaller file sizes, which results in shorter computation times during preprocessing and feature extraction. Therefore, they are useful for many real-time palm print applications [4].

\section{Palm print Biometric}

Palm print is one of the relatively new physiological biometrics due to its stable and unique characteristics. The rich texture information of palm print offers one of the powerful means in personal identification and verification. Biometric palm print recognizes a person based on the principal lines, wrinkles and ridges on the surface of the palm. These line structures are stable and remain unchanged throughout the life of an individual. The inner surface of the normally contains three flexion creases, secondary creases and ridges. The flexion creases are also called principal lines and secondary creases are called wrinkles. The flexion and the major secondary creases are formed between the 3rd and 5th months of pregnancy [5] and superficial lines appear after we born. Although the three major flexions are genetically dependent, most of other creases are not [6].

Even identical twins have different palm prints. These non-genetically deterministic and complex patterns are very useful in personal identification. Human beings were interested in palm lines for fortune telling long time ago. Scientists know that palm lines are associated with some genetic diseases including Down syndrome, Aarskog syndrome, Cohen syndrome and fetal alcohol syndrome [7]. Scientists and fortunetellers name the lines and regions in palm differently shown in Fig. 2 [8].

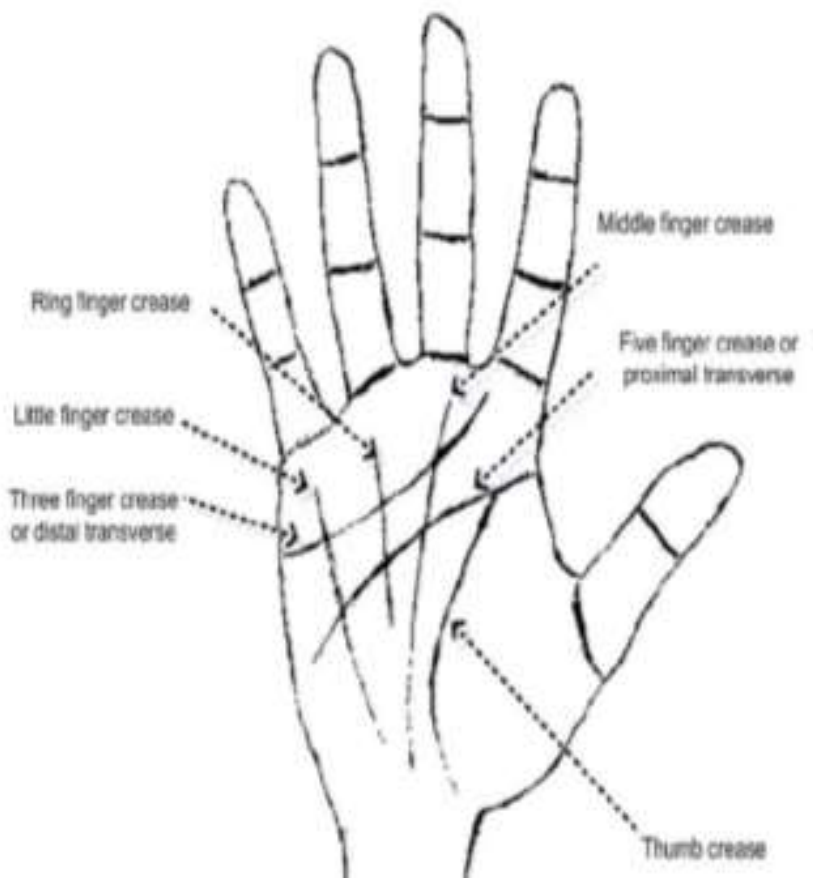

Fig. 2: Definitions of Palm lines and Regions from Scientists

\section{Face Recongnition}

Face recognition is the ability to establish a subject's identity based on facial characteristics. Face recognition has a wide range of applications, including Human-Computer interaction, Driver's license, National ID, Passports, Voter registration, Security system, Personal device logon, Desktop logon, Information security, Database security, Internet access, CCTV control and Suspect tracking and investigation. A face recognition system based on the age prediction is developed in this research. Face recognition is an important field of biometrics which is of great use in our day to day life. Be it the traditional uses in identification documents such as passports, driver's licenses, voter ID, etc., or its uses in recent years, where, face images are being increasingly used as additional means of authentication in applications such as credit/debit cards and in places of high security. But with age progression the facial features changes and the database needs to be 
updated regularly of the changes which is a tedious task. So we need to address the issue of facial aging and come up with a mechanism that identifies a person in spite of the aging.

\subsection{Age Dependent Face Recognition}

The system consists of two stages; age prediction and face recognition. In age prediction stage, eigenvectors and eigenfaces are calculated from the input image. New faces are projected onto the space expanded by eigenfaces and represented by weighted sum of the eigenfaces. These weights are used to identify the age of the faces. In face recognition stage, the predicted image will be searched to a certain age group of the database and all images within this age group would be considered as potential matches for the final level recognition. The age dependent face recognition system is developed based on 11 individual aging classes, which yields a great reduction time complexity in search space than searching the entire database. The algorithms that have been developed are tested on AT\&T, Yale, MORPH and FG-NET Face Databases.

\subsection{Age Prediction Accuracy Results}

In age prediction system, the accuracy rate is about 88 percent. The accuracy of the system can be analysed by the variation on the range of the age groups from the Database Faces of AT\&T Laboratories and face images database which are collected from UCSY (University of Computer Studies, Yangon) and the Internet. The larger errors occur at age 21 to 25 aging group and age 41 to 45 aging group. However, the overall prediction rate for all the experimental images is $88 \%$ by the proposed system. The results shown in Table I are tested using the database and the accuracy rate of the age prediction.

\begin{tabular}{c|cccc} 
TABLE I: Age Prediction System Performance for Testing Images \\
\hline Group & $\begin{array}{c}\text { Testing } \\
\text { Image }\end{array}$ & $\begin{array}{c}\text { Correctly } \\
\text { Predicted }\end{array}$ & Accuracy & Error \\
\hline 1 & 10 & 9 & $90 \%$ & $10 \%$ \\
2 & 10 & 9 & $90 \%$ & $10 \%$ \\
3 & 10 & 8 & $80 \%$ & $20 \%$ \\
4 & 10 & 9 & $90 \%$ & $10 \%$ \\
5 & 10 & 9 & $90 \%$ & $10 \%$ \\
6 & 10 & 9 & $90 \%$ & $10 \%$ \\
7 & 10 & 8 & $80 \%$ & $20 \%$ \\
8 & 10 & 9 & $90 \%$ & $10 \%$ \\
9 & 10 & 9 & $90 \%$ & $10 \%$ \\
10 & 10 & 9 & $90 \%$ & $10 \%$ \\
11 & 10 & 9 & $90 \%$ & $10 \%$ \\
\hline
\end{tabular}

\subsection{Experiment for Age Prediction}

In this system, the age groups are divided into 11 classes. The classes are: Class 1 (up to 15 years), Class 2 (16 to 20 years), Class 3 ( 21 to 25 years), Class 4 (26 to 30 years), Class 5 (31 to 35 years), Class 6 (36 to 40 years), Class 7 (41 to 45 years), Class 8 (46 to 50 years), Class 9 (51 to 55 years), Class 10 (56 to 60 years), Class 11 (more than 60 years).

Step One - Train the system: Twenty (20) images were selected for each class from the face database. The system was trained with these images using eigenface approach described the previous chapter, to derive the Training Feature Vector.

Step Two - Gather the testing images: Ten (10) images were selected for each class from the face database. The images were processed for classification by using the eigenface approach, to derive the Testing Feature Vector. 
Step Three - Classification: The PCA (Principle Component Analysis) classifier was used to enhance class separability. The minimum Euclidean distance of the Testing feature vector from the average distance of the three Training feature vectors was computed. The class with the minimum distance was defined as the winner. Thus the image was labelled with the age group of that particular class.

\subsection{System Performance and Evaluation}

The proposed system is evaluated by two values. The false acceptance rate, or FAR, is the measure of the likelihood that the proposed face recognition system will incorrectly accept an access attempt by an unauthorized user. FAR typically is calculated as the ratio of the number of false acceptances divided by the number of identification attempts. The false rejection rate, or FRR, is the measure of the likelihood that the proposed face recognition system will incorrectly reject an access attempt by an authorized user. FRR typically is calculated as the ratio of the number of false rejections divided by the number of identification attempts. The accuracy is the ratio of the number of correct recognition divided by the number of identification attempts.

FAR (False Accept Rate) is defined as follows:

$$
F A R=\frac{\text { no_accept }}{\text { no_test }} \times 100 \%
$$

FRR (False Reject Rate) is defined as follows:

$$
F R R=\frac{n o_{-} \text {reject }}{n o_{-} \text {test }} \times 100 \%
$$

Accuracy is calculated as:

$$
\text { Accuracy }=\frac{\text { no_true } \operatorname{Re} \text { sult }}{\text { no_test }} \times 100 \%
$$

- The no_test is denoted as the total number of tests.

- no_accept is denoted as the number of false accepted times.

- no_reject is denoted as the number of rejected times.

- no_trueResult is denoted as the total number of genuine matches.

For example, if "Htet Htet" enters "MaMa's" predicted age, presents her recognition data, and successfully matching as MaMa. This is classified as False Acceptance. The probability of this happening is referred to as False Acceptance Rate (FAR) [ stated as: percentage, fraction]. If "Htet Htet" enters her predicted age, presents her data to a face recognition system, and fails to match. This is classified as False Rejection. The probability of this happening is the False Rejection rate (FRR). This is because two people have similar enough biometric characteristics (a face) that the system finds a high degree of correlation between the users' template.

\subsection{Fusion of Face and Palm print}

At the time of fusion stage, face and palm print images will be acquired. Feature vectors are generated for each biometric trait and stored separately in the system database. At the time of identification, when user wants to prove his/her identity faces and palm print image will be captured using web camera. These images again will undergo image pre-processing and feature extraction stage. Euclidean distance formula is used to compute the distance between the eigen palm coefficients of the template and the query palm image. This will generate result 1. Euclidean distance formula is used to compute the distance between the template and query face image. Match result 2 is generated. All these two results will be passed to the fusion stage. Face images are represented using eign-coefficients, and the output of the face matcher is a distance result. Palm print images are represented using eigen palm-coefficients, and the output of the palm print matcher is a distance result. Finally, total result will be compared against the set threshold value. This will decide whether the person is correct or incorrect. 


\section{Conclusions}

This system presented the performance evaluation for personal identification that combines age dependent face recognition and palm print biometric. A two steps operation is used for this system, where the first step performs a prediction based on 11 individual aging classes and the second step performs the face recognition based on predicted age. The age prediction errors were due to the poor quality images, the lighting condition, and the large variation of pose and so on. The range of five-year age groups was used for age prediction. The complexity and processing time will be reduced by searching the matched face from predicted age group instead of searching the face from the database which contains 11 age groups. Experimental results show that both face recognition and age range estimation may be effectively performed with low computational effort. The accuracy of eigenface is also satisfactory (over $90 \%$ ) with frontal faces. The recognition errors (nearly 10\%) were due to the variation of pose, lighting, and so on. But most errors were caused due to the large changes of pose. The advantage of the eigenface method is the system's speed and efficiency. The eigenface approach reduces the amount of data. The personal identification system can achieve good performance in terms of speed and accuracy using palm print and face recognition.

\section{Acknowledgement}

I would like to thank my husband for his valuable advice, unconditional love and support without which this journey would not have been possible.

\section{References}

[1] S. NEC Automated Palmprint Identification System http://www.necmalaysia.com.my/Solutions/PID/products/ppi.html

[2] N. Duta, A.K. Jain and K.V. Mardia, "Matching of palm prints", Pattern Recognition Letters, vol. 23, no. 4, pp. 477 486, 2002. http://dx.doi.org/10.1016/S0167-8655(01)00179-9

[3] W. Shu and D. Zhang, "Automated personal identification by palmprint", Optical Engineering, vol. 38, no.8, pp. 23592362, 1998.

http://dx.doi.org/10.1117/1.601756

[4] David Zhang, Wai-Kin Kong and Jane You, "On-Line Palmprint Identification".

[5] M. Cannon, M. byrne, D. Cotter, P.Sham, C. Larkin, E. O'Callaghan, "Futher evidence for anomalies in the handprints of patients with schizophrenia : a study of secondary creases". Schizophrenia Research, vol.13, pp. 179-184, 1994.

http://dx.doi.org/10.1016/0920-9964(94)90100-7

[6] (A. Kong, d. Zhang and G. Lu, "A study of identical twins palm print for personal verification”, Pattern Recognition, vol. 39, no. 11, pp. 2149-2156, 2006. http://dx.doi.org/10.1016/j.patcog.2006.04.035

[7] The National Fragile X Foundation http://www.nfxf.org/html/checklist.htm

[8] L.S. Penrose, "Fingerprints and palmistry", The Lancet, vol. 301, no 7814, pp.1239-1242, 1973. http://dx.doi.org/10.1016/S0140-6736(73)90543-6

[9] Hlaing Htake Khaung Tin, "Facial Extraction and Lip Tracking Using Facial Point", International Journal of Computer Science and Engineering and Information Technology (IJCSEIT), vol.1, no.1, April 2011.

[10] Hlaing Htake Khaung Tin and Myint Myint Sein, "Fast Facial Recognition System", IEEE The proceeding of the $8^{\text {th }}$ International Joint Conference on Computer Science and Software Engineering (JCSSE 2011), May 2011.

[11] Hlaing Htake Khaung Tin and Myint Myint Sein, "Effective Method of Age Dependent Face Recognition", The proceeding of International Conference on Computer Science and Informatics (ICCSI 2011), June 2011.

[12] Hlaing Htake Khaung Tin and Myint Myint Sein, "Effective Method of Age Dependent Face Recognition", International Journal of Computer and Informatics (IJCSI), vol.1, issue.1, June 2011.

[13] Hlaing Htake Khaung Tin and Myint Myint Sein, "Race Identification from Face Images", The proceeding of International Conference on Advances in Computer Engineering (ACE 2011), August 2011. 
[14] Hlaing Htake Khaung Tin and Myint Myint Sein, "Race Identification from Face Images", International Journal on Information Technology (IJIT), vol.1, issue.2, August 2011.

[15] Hlaing Htake Khaung Tin and Myint Myint Sein, "Robust Method of Age Dependent Face Recognition", IEEE the 4th International Conference on Intelligent Networks and Intelligent Systems (ICINIS 2011), November 2011.

[16] Hlaing Htake Khaung Tin and Myint Myint Sein, "Developing the Age Dependent Face Recognition System", International Journal of Intelligent Engineering and Systems (IJIES), vol.4, issue.4, December 2011.

[17] Hlaing Htake Khaung Tin and Myint Myint Sein, "Aging Groups Classification based on Facial Feature", The proceeding of International Conference on Information Technology Management Issues (ICITMI 2011), September 2011.

[18] Hlaing Htake Khaung Tin and Myint Myint Sein, "Aging Group Classification based on Facial Feature”, Journal of Information Systems : New Paradigms (JISNP), vol.1, no.1, September 2011.

[19] Hlaing Htake Khaung Tin, "Perceived Gender Classification from Face Images", International Journal of Modern Education and Computer Science (IJMECS), vol.4, no.1, February 2012.

[20] Hlaing Htake Khaung Tin, "Robust Algorithm for Face Detection in Color Images", International Journal of Modern Education and Computer Science (IJMECS), vol.4, no.2, March 2012.

[21] Hlaing Htake Khaung Tin, "Subjective Age Prediction of Face Images Using PCA", International Journal of Information and Electronics Engineering (IJIEE), vol.2, no.3, May 2012.

[22] Hlaing Htake Khaung Tin, "Gender and Age Estimation Based on Facial Images", International Journal : ACTA TECJNICA NAPOCENSIS Electronics and Telecommunications, vol. 52, no.3, 2011.

[23] Hlaing Htake Khaung Tin and Myint Myint Sein, "Feature based Age Prediction for Face Recognition", The 5th Parallel and Soft Computing (PSC 2010), December 2010.

[24] Hlaing Htake Khaung Tin and Myint Myint Sein, "Automatic Aging Simulation of the Human Face", The 9th International Conference on Computer Applications (ICCA 2011), May 2011.

[25] Hlaing Htake Khaung Tin and Myint Myint Sein, "Removal of Noise Reduction for Image Processing", The $6^{\text {th }}$ Parallel and Soft Computing (PSC 2011), December 2011.

[26] Hlaing Htake Khaung Tin and Myint Myint Sein, "Automatic Age Prediction of Aging Effects on Face Images", The $10^{\text {th }}$ International Conference on Computer Applications (ICCA 2012), February 2012.

[27] Hlaing Htake Khaung Tin, "Personal Identification and Verification using Palm Print Biometric", The $3^{\text {rd }}$ International Conference on Advancement of Engineering (ICAE 2012), December 2012.

[28] Hlaing Htake Khaung Tin, "Personal Identification and Verification using Palm Print Biometric", International Journal of Latest Technology in Engineering Management and Applied Science (IJLTEMAS), vol. 1, issue. X, December 2012.

[29] Hlaing Htake Khaung Tin, "Effective Method of Face Recognition and Palmprint Biometrics for Personal Identification", International Journal of Advanced and Innovative Research (IJAIR), December 2012.

[30] Hlaing Htake Khaung Tin, Myint Myint Sein "How Old Are You?: Age Prediction using Eigen Face", International Conference on Science and Engineering (ICSE 2013), December 2013.

[31] Hlaing Htake Khaung Tin, Myint Myint Sein "Real time age prediction and gender classification", The First International Conference on Energy Environment and Human Engineering (ICEEHE 2013), December 2013.

[32] Hlaing Htake Khaung Tin, Myint Myint Sein "Automatic Race Identification from Face Images in Myanmar", The First International Conference on Energy Environment and Human Engineering (ICEEHE 2013), December 2013.

[33] Hlaing Htake Khaung Tin "What is your race?:Ethnicity and Race Identification in Myanmar", International Conference on Research and Scientific Innovation (ICRSI 2013), December 2013. 